\title{
Amiodarone management of junctional ectopic tachycardia after cardiac surgery in children
}

\author{
P Raja, R E Hawker, A Chaikitpinyo, S G Cooper, K C Lau, G R Nunn, T B Cartmill,
} G F Sholler

\footnotetext{
Adolph Basser Institute of Cardiology, Royal Alexandra Hospital for Children, Camperdown, Sydney, Australia P Raja

R E Hawker

A Chaikitpinyo

$S$ G Cooper

KC Lau

G R Nunn

T B Cartmill

G F Sholler

Correspondence to: Dr R E Hawker, Adolph Basser Institute of Cardiology, The Royal Alexandra Hospital for Children, Camperdown, Children, Camperdown,
Sydney, Australia 2050. Accepted for publication 28 March 1994
}

\begin{abstract}
Objective-To assess the effectiveness and safety of amiodarone in the treatment of junctional ectopic tachycardia (JET) after open heart surgery in children. Patients-Between January 1990 and December 1991, 16 consecutive patients aged 6 days to 14 years with JET associated with significant haemodynamic impairment after cardiopulmonary bypass were treated with amiodarone as the principal antiarrhythmic drug.

Interventions-Amiodarone $5 \mathrm{mg} / \mathrm{kg}$ was administered intravenously over one hour and the same dose was subsequently infused over 12 hours. This was reviewed every 12 hours and repeated as necessary until a satisfactory heart rate and stable haemodynamics were achieved. Atrial pacing was used whenever possible to provide atrioventricular synchrony.

Results-Except for one patient with a JET rate of $160 / \mathrm{min}$, the maximum JET rate ranged from $180 / \mathrm{min}$ to $245 / \mathrm{min}$ with a mean(SD) of 200 (20)/min. After amiodarone, the heart rates reduced to a mean(SD) of 170 (20), 164 (27), 158 (27), 157 (24), and 153 (19)/min at two, four, eight, 12, and 24 hours respectively. A reduction in tachycardia rate allowing atrial pacing was achieved in 10 patients by two hours. Haemodynamic variables improved in most patients with an increase in mean systolic blood pressure by an average of $15 \mathrm{~mm} \mathrm{Hg}$ and a decrease in atrial filling pressures by an average of $3.5 \mathrm{~mm} \mathrm{Hg}$ at four hours after amiodarone administration. There were three deaths: one was a moribund patient who died soon after the onset of JET and the other two deaths were not directly related to JET.

Complications-Late bradycardia with hypotension was recorded in one patient. Asymptomatic late sinus bradycardia was seen in several others.

Conclusions-Amiodarone can be used safely and effectively to control JET with haemodynamic improvement in most patients. The addition of atrial pacing confers the advantage of atrioventricular synchrony.
\end{abstract}

(Br Heart f 1994;72:000-000)

Junctional ectopic tachycardia (JET) occurring after cardiac surgery in children is a serious arrhythmia that is associated with considerable morbidity and mortality. ${ }^{1-3}$ Also known as His bundle tachycardia, ${ }^{4}$ it is typically characterised by atrioventricular dissociation and rapid junctional rates which may lead to significant haemodynamic impairment. Success with pharmacological treatment of postoperative JET in infants and children has been limited. ${ }^{56}$ The reported efficacy of amiodarone for congenital JET, together with its minimal negative inotropic effect, led us to use it for the treatment of postoperative JET. To provide atrioventricular synchrony atrial pacing was used whenever possible. This paper reports our experience with this approach.

\section{Patients and methods}

PATIENTS

A retrospective study of the medical records of 790 infants and children returning to the cardiac intensive care unit after heart surgery between January 1990 and December 1991 identified 16 patients in whom JET had been treated with amiodarone according to our protocol. Their ages ranged from 6 days to 14 years with a median age of 7 months. Preoperatively none of these patients had Wolff-Parkinson-White syndrome or rhythm abnormalities. The table lists the lesions repaired. Two patients developed JET at reoperation for residual ventricular septal defect (one after initial atrioventricular septal defect repair and the other after truncus arteriosus repair).

All operations had been performed under cardiopulmonary bypass with bypass times ranging from 160 to 180 minutes. The table lists the associated inotropic support.

\section{DEFINITION AND DIAGNOSIS}

Diagnosis of JET was made from the surface electrocardiogram (ECG) in most patients and confirmed when necessary by atrial electrogram (fig 1). Junctional ectopic tachycardia was characterised by (a) an incessant tachycardia with a heart rate greater than the 98th centile for age, (b) a narrow QRS or wide QRS in the presence of surgically induced bundle branch block, and $(c)$ the presence of atrioventricular dissociation with a ventricular rate greater than the atrial rate ${ }^{4}$ or retrograde $1: 1$ ventriculo-atrial conduction.

\section{MANAGEMENT}

Treatment with amiodarone was indicated when the JET rate exceeded $170 / \mathrm{min}$ and was 
Clinical data of 16 patients with junctional ectopic tachycardia

\begin{tabular}{|c|c|c|c|c|c|c|}
\hline $\begin{array}{l}\text { Patient } \\
\text { No }\end{array}$ & Age & Diagnosis & Operation & Inotrope & Vasodilator & $\begin{array}{l}\text { Maximum } \mathcal{F E T} \\
\text { rate (beats } / \mathrm{min} \text { ) }\end{array}$ \\
\hline 1 & 5 months & VSD & $\mathbf{P}$ & $\mathrm{D}$ & $\mathbf{N}$ & 210 \\
\hline 2 & 13 months & Res VSD (post-AVSD) & $\mathbf{P}$ & $\mathrm{D}$ & $\mathbf{N}$ & 180 \\
\hline 3 & 2 months & VSD & $\mathbf{P}$ & D & $\mathrm{N}$ & 190 \\
\hline 4 & 10 months & AVSD & $\mathrm{C}$ & $\mathrm{D}, \mathrm{NE}$ & $\mathrm{Ph}$ & 220 \\
\hline 5 & 8 months & AVSD & $\mathrm{C}$ & $\mathrm{D}, \mathrm{A}, \mathrm{NE}$ & $\mathrm{Ph}$ & 190 \\
\hline 6 & 11 months & Truncus & $\mathrm{C}$ & $\mathrm{D}, \mathrm{A}, \mathrm{NE}$ & $\mathrm{N}, \mathrm{Ph}$ & 180 \\
\hline 7 & 14 years & AVSD/PA/TAPVD, shunts & Glenn, C TAPVD & $\mathrm{D}, \mathrm{A}$ & $\mathbf{N}$ & 190 \\
\hline 8 & 1 month & Res, VSD (post truncus) & $\mathrm{P}$ & $\mathrm{D}, \mathrm{A}, \mathrm{NE}$ & $\mathrm{N}, \mathrm{Ph}$ & 200 \\
\hline 9 & 32 months & TET & $\mathrm{C}$ & $\mathrm{D}, \mathrm{A}$ & $\mathbf{N}$ & 200 \\
\hline 10 & 15 months & TET & $\mathrm{C}$ & $\mathrm{D}, \mathrm{A}, \mathrm{NE}$ & $\mathrm{N}, \mathrm{Ph}$ & 180 \\
\hline 11 & 4 months & VSD & $\mathbf{P}$ & D & N & 190 \\
\hline 12 & 6 months & AVSD & $\mathrm{C}$ & $\mathrm{D}, \mathrm{A}, \mathrm{NE}$ & $\mathrm{Ph}$ & 210 \\
\hline 13 & 3 weeks & TGA/VSD & $\mathrm{s}$ & $\mathrm{D}, \mathrm{A}$ & $\mathrm{N}$ & 220 \\
\hline 14 & 5 months & AVSD & C & $\mathrm{D}, \mathrm{A}$ & $\mathrm{N}$ & 190 \\
\hline 15 & 26 months & PAVSD & C & $\mathrm{D}, \mathrm{A}$ & $\mathrm{N}$ & 160 \\
\hline 16 & 5 days & TGA & $S$ & $\mathrm{D}, \mathrm{NE}$ & $\mathrm{Ph}$ & 245 \\
\hline
\end{tabular}

Abbreviations: AVSD = atrioventricular septal defect; VSD = ventricular septal defect (Res = residual); Truncus = common arterial trunk; TET = tetralogy of Fallot; TGA = transposition of great arteries; $\mathrm{PA}=$ pulmonary atresia; $\mathrm{P}=$ patch closure $\mathrm{C}=$ correction; $\mathrm{D}=$ dopamine; $\mathrm{A}=$ adrenaline; $\mathrm{NE}=$ noradrenaline; $\mathrm{N}=$ nitroprusside; and $\mathrm{Ph}=$ phenoxybenzamine $\mathrm{TAPVD}=$ Total anomalous pulmonary venous drainage

associated with important haemodynamic impairment. This particular rate was selected because (a) although sinus rates in excess of this are well tolerated in infants, atrioventricular dissociation is not and (b) the standard pacemaker units in our intensive care unit have a maximum rate of $180 / \mathrm{min}$. Twelve patients with junctional rates around $170 / \mathrm{min}$ (or higher for brief periods) were not given amiodarone because haemodynamic function was satisfactory either alone or with atrial pacing. During the period of study there were for comparison 57 patients with junctional rates $<150 / \mathrm{min}$ who were managed with atrial pacing if this could be shown to improve the haemodynamics. Deliberate hypothermia was not used as a mode of treatment in these patients, but hyperthermia associated with low cardiac output was always vigorously treated. Three patients received digoxin initially. One had a junctional rate of $240 / \mathrm{min}$ with some slowing after digoxin. Amiodarone was started one hour later because of a secondary increase in the heart rate. A second patient was considered to have atrial tachycardia of the re-entrant type because of a rate of $240 / \mathrm{min}$ and no evidence of atrioventricular dissociation. $\mathrm{He}$ initially responded to digoxin, reverting to sinus rhythm at a rate of $180 / \mathrm{min}$. Junctional ectopic tachycardia developed the next day and amiodarone was started. In the third patient, digoxin was given as the initial drug in view of the low cardiac output and hypotension in the presence of a relatively slow JET rate of $160 / \mathrm{min}$. This patient had ventricular wires but no atrial wires and continuous transoesophageal pacing was used to provide atrioventricular synchrony. Amiodarone was started the next day to prevent a further increase in JET rate. Thus this patient differed from the rest of the series in that amiodarone was used prophylactically to prevent an increase in JET rate with possible further depression of cardiac output when atrial pacing wires were not available.

No other antiarrhythmic drugs were used in these patients. Electrolytes, acid-base balance, and calcium concentrations were closely monitored and abnormalities were treated accordingly. In this group of patients with unstable circulation multiple changes to inotropes and vasodilators were made. The retrospective study did not, however, identify any acute changes in drugs necessitated by adverse reactions to amiodarone.

AMIODARONE ADMINISTRATION

Amiodarone $5 \mathrm{mg} / \mathrm{kg}$ was initially given intravenously over one hour. The same dose was then given as an infusion over 12 hours. This
Figure 1 Atrial electrocardiogram showing atrioventricular dissociation with atrial spikes (a) and ventricular spikes $(v)$ at rates of $120 /$ min and 195/min respectively.

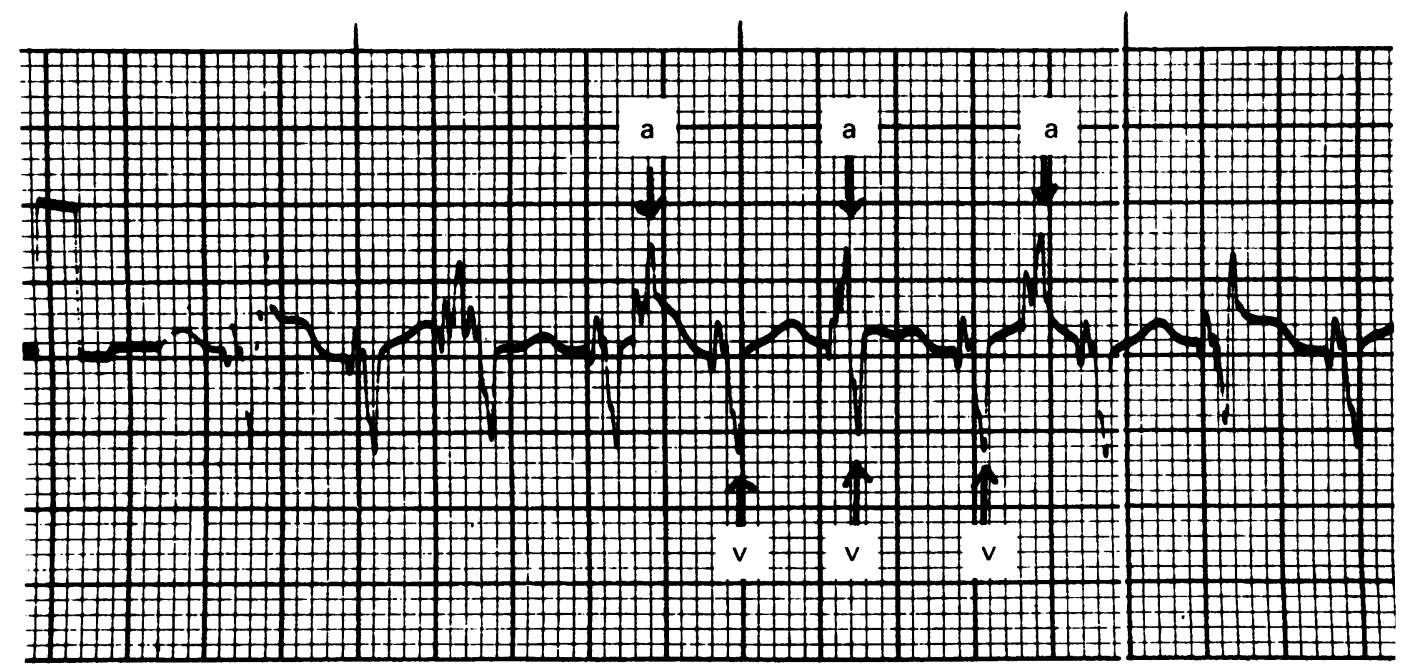


Figure 2 Blood pressure (BP) at $0(\bullet)$ and $4 h(-)$ after amiodarone.

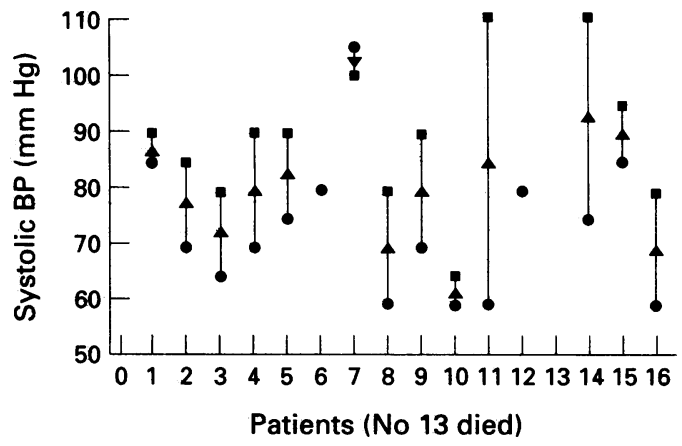

was reviewed every 12 hours and repeated as necessary until slowing of the JET rate to a level where atrial pacing was consistently possible (usually less than $170 / \mathrm{min}$ ) was achieved. The infusion was stopped when sinus rhythm returned or when sufficient slowing of the JET rate occurred to allow stable haemodynamic function. Amiodarone was restarted if the JET rate increased or overriding of the atrial pacemaker occurred, or both. Atrial pacing was continued whenever atrial capture was not haemodynamically beneficial. The duration of treatment ranged from one hour to 10 days (median 24 hours). Amiodarone by mouth was not required. Because of these short courses of treatment, we did not think that measurement of blood concentrations was warranted clinically.

PATIENT DATA

The intensive care unit monitoring charts were reviewed to determine the maximum JET rates, systolic blood pressure, central venous pressure, or left atrial pressure before amiodarone administration. The heart rate was measured two, four, eight, 12 , and 24 hours after amiodarone administration. The blood pressure and central venous pressure (or left atrial pressure) were documented at four hours after amiodarone administration.

\section{Results}

JET started 0 to 78 hours (median six hours) after return of the patients to the intensive care unit. In 11 of 16 patients it occurred within 12 hours. Except for one patient, the maximum JET rate ranged from 180 to $245 / \mathrm{min}$, with a mean (SD) of $198 / \mathrm{min}(20)$. After amiodarone, the heart rates reduced to a mean (SD) of 170 (20), 164 (27), 158 (27),

Figure 3 Central venous pressure (CVP)/left atrial pressure (LAP) at $0(\bullet)$ and $4 h(\square)$ after amiodarone.

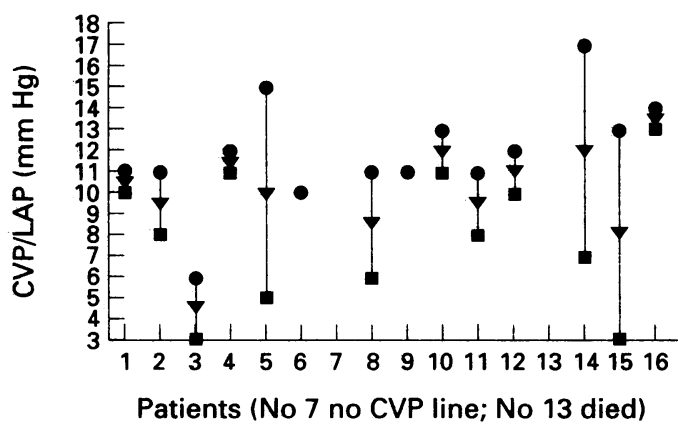

157 (24), and $153(19) / \mathrm{min}$ at two, four, eight, 12 , and 24 hours respectively (fig 2 ). Of the 15 patients who survived the tachycardia, a reduction to a rate at which atrial pacing was possible was achieved in 10 patients by two hours; only one of 15 patients had a heart rate more than $180 / \mathrm{min}$ at eight hours.

Prolonged atrial pacing was used in 10 of the 16 patients. Of the other six patients, two returned to sinus rhythm three and five hours after amiodarone and pacing was used only briefly; in one with alternating nodal and sinus rhythm (rate 120/min) haemodynamic function was satisfactory and not improved with pacing; one could not be captured; one died soon after the onset of JET; and in one patient significant bradycardia with hypotension developed eight hours after amiodarone was started and demand ventricular pacing was begun as he had no atrial wires.

Haemodynamic variables were assessed four hours after amiodarone administration. Blood pressure increased in most patients (fig 2), with the systolic blood pressure increasing from a mean (SD) of 75 (12) $\mathrm{mm} \mathrm{Hg}$ to 90 (11) $\mathrm{mm} \mathrm{Hg}$, before and four hours after amiodarone respectively (fig 2). There was also a concomitant reduction of atrial filling pressures in most patients (fig 3). The atrial pressures before and four hours after amiodarone were $12.0(2.5)$ and $8.5(3.0) \mathrm{mm} \mathrm{Hg}$ respectively. The interval before the return of sinus rhythm ranged from three to 120 hours (median 72 hours). Before complete return to sinus rhythm a period of alternating nodal and sinus rhythm often occurred. Amiodarone was continued for 10 days in one patient because of multiple atrial extra systoles.

\section{COMPLICATIONS}

There was no significant decrease in blood pressure during the initial infusion of amiodarone. The severe bradycardia that developed in one patient eight hours after starting amiodarone could have had other causes and was easily managed by pacing and stopping the amiodarone. Late sinus bradycardia occurred in some patients during the recovery period without significant haemodynamic impairment. The QT intervals could not be reliably measured from monitored rhythm strips at fast rates, particularly if there was bundle branch block. No impression of QT prolongation was obtained and torsades de pointes did not occur, however.

\section{MORTALITY}

Three patients died. The first was a 1 month old infant after arterial switch repair of transposition of the great arteries. His condition had been unstable after return to the intensive care unit and JET developed at 12 hours. He rapidly deteriorated and could not be resuscitated. The second was an infant aged 6 months with Down's syndrome in whom JET developed eight hours after repair of an atrial septal defect. JET was controlled with amiodarone and atrial pacing for three days. Three hours after late closure of the sternum, he arrested suddenly and died. The third was a 
patient who had truncus arteriosus repair initially and developed JET at reoperation for residual ventricular septal defect. He subsequently died after a third operation was required for aortic valve replacement. Amiodarone was not identified as contributing significantly to death in any of these three patients.

\section{Discussion}

Junctional ectopic tachycardia after cardiac surgery may be a life threatening arrhythmia in infants, although it is mostly well tolerated in adult patients. The incidence is unknown but retrospective review of 154 children undergoing open heart surgery from 1983 to 1984 by Grant et al identified 12 cases of JET with significant haemodynamic impairment. ${ }^{1}$ Chaikitpinyo et al, from our unit, observed junctional tachycardia at a rate above $150 / \mathrm{min}$ in $7 \%$ of 395 patients returning to the intensive care unit after open heart surgery. ${ }^{8}$ Till and Rowland reported 11 patients in a two year nine month review period. ${ }^{4}$

This arrhythmia is thought to result from enhanced automaticity within the bundle of His. ${ }^{169}$ Cellular damage associated with the operation around the region of the bundle of His has been implicated in its pathogenesis. It occurs in few infants and children having an operation in this region, however. Delay after depolarisation has also been proposed as a possible mechanism. ${ }^{9}$ Occasionally, JET can occur in patients where the operation was remote from the bundle of $\mathrm{His},{ }^{5}$ suggesting other indirect mechanisms; we have postulated that stretching of the node may alter its electrical behaviour. In our series, 14 of 16 patients had an operation near the bundle of His and two patients had an operation remote from it (patients 7 and 16).

Physical measures have been used to attempt control of JET. Hypothermia has been used to slow the heart rate by depressing automaticity. ${ }^{451011}$ Balagi et al reduced the mean junctional rate from 211 to 146 in seven mostly postoperative patients by lowering their core temperature to $32-34^{\circ} \mathrm{C} .^{11}$ Antiarrhythmic drugs were not given in four of these. Improved blood pressure and urine output was documented but three patients nevertheless died. As in our series and that of Till and Rowland, ${ }^{4}$ the relative contributions to death of the arrhythmia in itself, the underlying cardiac disorder, and multiple perioperative factors is hard to determine.

Augmentation of cardiac output by establishing atrioventricular synchrony was well documented by Till and Rowland, who reported the use of a combination of hypothermia, amiodarone, and other drugs to slow the junctional rate. ${ }^{4}$ They achieved immediate benefit by coupling atrial stimulation to the preceding $\mathrm{His}$ bundle ectopic focus. We agree with this group that early recognition and immediate treatment will maximise salvage and we believe that the staged protocol reported earlier by Sholler $e t$ al has potential disadvantages. ${ }^{5}$
The postoperative and congenital forms of JET have generally been refractory to conventional antiarrhythmic drugs. Grant et $a l^{1}$ reported the successful use of digoxin. A favourable response was not seen by others, ${ }^{512}$ however. Propranolol and verapamil are generally ineffective, ${ }^{512}$ and their use is also limited by their depressant effect on the myocardium. Newer class 1c drugs such as propafenone $^{12}$ and flecainide ${ }^{12-14}$ have had some success but their known negative inotropic effects limit their use in the critical postoperative period. Waldo et $a l^{15}$ and Slysmans et al ${ }^{16}$ described paired ventricular pacing as a means of reducing the heart rate, and surgical ablation of the bundle of His has been reported as a last resort procedure. ${ }^{17}$

Amiodarone hydrochloride is a class 3 antiarrhythmic drug that prolongs the repolarisation and refractory period of atrial, nodal, and ventricular tissues, thereby giving it a wide range of activity. ${ }^{18}$ Its effect in JET may relate to its inhibition of abnormal automaticity, and the increase in the refractory period of the atrioventricular node and His-Purkinje system. When given intravenously, a loading dose is required because of the rapid disappearance of the drug from plasma during the distribution phase, which appears to be large. Continuous infusion is then necessary to sustain steady state plasma drug concentrations of about $1 \mu \mathrm{g} / \mathrm{ml} .{ }^{19}$ The drug is metabolised to desethylamiodarone, the activity of which is uncertain, and excreted through the liver. The dose regimen was based on the manufacturer's recommendation, though lower maintenance doses were given. Hypotension was not definitely recognised after the initial infusion, but we were prepared to use volume expansion rather than give further inotropes that may increase the heart rate. Although prolongation of the QT interval is expected with long term administration by mouth, this was not recognised in our patients receiving amiodarone intravenously.

Amiodarone has been used to treat congenital JET ${ }^{20-22}$ intravenously in the acute phase and by mouth to prevent recurrence. Villain $e t$ $a l$, in a multicentre study, reported 14 patients with congenital JET who were treated with amiodarone with eight successes and three partial successes. ${ }^{20}$ Till et al had a subset of seven patients who were given amiodarone postoperatively, with reduction in the tachycardia rate in four. ${ }^{3}$

Over the two year period of our review, amiodarone was used as the principal drug to control JET and was supplemented with atrial pacing. Two thirds of the patients had reduction of JET rate to less than $180 / \mathrm{min}$ by two hours and nearly all patients within four hours. It was then possible to use overdrive atrial pacing to provide atrioventricular synchrony. We found that the junctional rate was reduced as the myocardium recovered and the dose of inotropic drugs decreases, whether or not amiodarone has been used. We would therefore prefer that atrial pacing wires are available in all patients who have had an operation under cardiopulmonary bypass, but 
transoesophageal pacing was possible in one patient when they were not.

Recovery to sinus rhythm seemed to be spontaneous and probably not directly influenced by amiodarone. Our rationale for treatment, however, was the reduction of the JET rate first and then the production of atrial conducted rhythm by atrial pacing. Further improvement in cardiac and metabolic function allows spontaneous return of sinus rhythm, which generally occurs within three to seven days. Because of the long time needed for this drug to reach equilibrium, any correlation of blood concentration with clinical response would probably be difficult to establish.

No serious complications were encountered. When compared with other series of patients with JET ${ }^{1457}$ the mortality in our group of patients was relatively low and could not be attributed directly to either adverse drug reaction or failure of the treatment.

We believe that amiodarone is an effertive and safe method of controlling JET after cardiac surgery in children.

1 Grant JW, Serwer GA, Armstrong BE, Oldham HN, Anderson PAW. Junctional tachycardia in infants and children after open heart surgery for congenital hear disease. Am F Cardiol 1987;59:1216-8.

2 Krongrad E. Postoperative arrhythmias in patients with congenital heart disease. Chest 1984;85:107-13.

3 Till JA, Rowland E, Rigby M. His bundle tachycardia: an important cause of postoperative morbidity and mortality. In: Crupi G, Parenzan I, Anderson RH, eds. Perspectives in paediatric cardiology. Vol. 2. New York: Futura, 1990: in paediati $269-72$.

4 Till JA, Rowland E. Atrial pacing as an adjunct to the management of post surgical His bundle tachycardia. $B$ Heart $¥ 1991 ; 66: 225-9$.

5 Sholler GF, Walsh EP, Mayer JE, Saul JP, Gamble WJ, Lang $P$. Evaluation of staged treatment protocol for postoperative rapid junctional tachycardia [abstract] Circulation 1988;78(suppl 11):597.
6 Garson A, Gillette PC. Junctional ectopic tachycardia in children: electrocardiography, electrophysiology and pharmacological response. Am $f$ Cardiol 1979;44: 298-302.

7 Gillette PC. Diagnosis and management of postoperative junctional ectopic tachycardia. Am Heart $\mathcal{f} 1989 ; 118$ : 192-4.

8 Chaikitpinyo A, Hawker RE, Cartmill TB. Arrhythmia after open heart surgery in infants and children [abstract]. In: Proceedings of 9th ASEAN Congress in Cardiology; 1992:397.

9 Rosen MR, Fisch C, Hoffman BF, Danilo P, Lovelace DE, Knoebel SB. Can accelerated atrioventricular junctional escape rhythms be explained by delayed after depolarisations? Am $\mathcal{F}$ Cardiol 1980;45:1272-84.

10 Bash S, Shah J, Albers WH, Geiss DM. Hypothermia for the treatment of post surgical greatly accelerated junctional ectopic tachycardia. $\Im \mathrm{Am}$ Coll Cardiol 1987;10: tonal -9.

11 Balaji S, Sullivan I, Deanfield J, James I. Moderate hypothermia in the management of resistant automatic tachycardias in children. Br Heart 7 1991;66:221-4.

12 Garson A, Moak JP, Smith RT, Norton JB. Usefulness of intravenous propafenone for control of postoperative junctional ectopic tachycardia. Am $\mathcal{F}$ Cardiol 1987;59: $1422-4$.

13 Kuch KH, Kunze KP, Schluter M, Duckeck W. Encainide versus flecainide for chronic atrial tachycardia and junctional ectopic tachycardia. Am 7 Cardiol 1988;62: $37 \mathrm{~L}-44 \mathrm{~L}$.

14 Wren C, Campbell RWF. His bundle tachycardiaarrhythmogenic and antiarrhythmic effects of therapy. Eur Heart $\mathcal{f}$ 1987;8:647-50.

15 Waldo AL, Krongrad E, Kupersmith J, Levine OR, Bowman FO, Hoffman BF. Ventricular paired pacing to control ventricular heart rate following open heart surgery. Circulation 1976;53:176-81.

16 Slysmans T, Moulin D, Jaumin P, Rubay J, Vliers A DeJonghe $D$. Ventricular paired pacing to control DeJonghe $\mathrm{D}$. Ventricular paired pacing to control surgery in a child. Intensive Care Med 1989;15:203-5.

17 Braunstein PW, Sade RM, Gillette PC. Life threatening postoperative junctional ectopic tachycardia. Ann Thorac Surg 1992;53:726-8.

18 Katritsis D, Cam AJ. Amiodarone in long term prophylaxis. Drugs 1991;41 (suppl 2):54-66.

19 Wilkinson LL, et al. Cordarone X (Reckitt \& Colman Pharmaceuticals). In: MIMS Annual 1989 (Australian edition) Sydney: IMS Publishing: 2-52.

20 Villain E, Vetter VL, Garcia JM, Herre J, Cifarelli A, Garson A. Evolving concepts in the management of congenital junctional ectopic tachycardia. A multicenter study. Circulation 1990;81:1544-9.

21 Coumel P, Fidelle J. Amiodarone in the treatment of cardiac arrhythmias in children: 135 cases. Am Heart 7 diac arrhythmias

22 Garson A, Gillette PC, et al. Amiodarone treatment of critical arrhythmias in children and young adults. $₹ \mathrm{Am} \mathrm{Coll}$ Cardiol 1984;4:749-55. 